\title{
Trichilemmal Carcinoma from Proliferating Trichilemmal Cyst on the Posterior Neck
}

\author{
Ui Geon Kim ${ }^{1}$, \\ Dong Bee Kook ${ }^{1}$, \\ Tae Hun Kim², \\ Chung Hun Kim ${ }^{1}$ \\ Departments of ${ }^{1}$ Plastic and Reconstructive \\ Surgery and ${ }^{2}$ Pathology, Bundang CHA \\ Medical Center, CHA University School of \\ Medicine, Seongnam, Korea
}

No potential conflict of interest relevant to this article was reported.

\begin{abstract}
Trichilemmal cysts are common fluid-filled growths that arise from the isthmus of the hair follicle. They can form rapidly multiplying trichilemmal tumors-, also called proliferating trichilemmal cysts, which are typically benign. Rarely, proliferating trichilemmal cysts can become cancerous. Here we report the case of a patient who experienced this series of changes. The 27 -year-old male patient had been observed to have a $1 \times 1 \mathrm{~cm}$ cyst 7 years ago. Eight months prior to presentation at our institution, incision and drainage was performed at his local clinic. However, the size of the mass had gradually increased. At our clinic, he presented with a $5 \times 4 \mathrm{~cm}$ hard mass that had recurred on the posterior side of his neck. The tumor was removed without safety margin, and the skin defect was covered with a split-thickness skin graft. The pathologic diagnosis was a benign proliferating trichilemmal cyst. The mass recurred after 4months, at which point, a wide excision (1.3-cm safety margin) and split-thickness skin graft were performed. The biopsy revealed a trichilemmal carcinoma arising from a proliferating trichilemmal cyst. This clinical experience suggests that clinicians should consider the possibility of malignant changes when diagnosing and treating trichilemmal cysts.
\end{abstract}

Keywords: Epidermal cyst / Hair disease / Skin neoplasms / Skin appendage

\section{INTRODUCTION}

Trichilemmal cysts are keratin-filled cysts derived from the exposed outer root sheath of hair follicles [1]. Trichilemmal cysts are typically benign but can form rapidly multiplying trichilemmal tumors, also called proliferating trichilemmal cysts (PTCs) [2]. Brownstein and Arluk [1] reported that trauma and inflammation can induce this change. PTCs arise in areas with high hair follicle density (e.g., scalp) in more than $90 \%$ of patients, but can also occur on the back, vulva, nose, mons pubis, buttock, wrist, chest, or elbow. PTCs are five times more common in women than in men. Although the average patient age is 65 years [3], there is a case report of PTCs developing on the arm of an 18-year-old

Correspondence: Chung Hun Kim

Department of Plastic and Reconstructive Surgery, Bundang CHA Medical Center, CHA

University School of Medicine, 59 Yatap-ro, Bundang-gu, Seongnam 13496, Korea

E-mail: chkimps@cha.ac.kr

Received October 27, 2016 / Revised February 8, 2017 / Accepted February 8, 2017 woman [4]. The masses usually range in size from $<1$ to $10 \mathrm{~cm}$, but a 25-cm PTC on the scalp has been reported [5].

There are several documented cases of PTCs undergoing carcinomatous transformation. Sudden enlargement of long-lasting lesions and exophytic expansion suggest malignant transformation [3]. Saida et al. [2] described three stages in this oncological development: the trichilemmal cyst is a tumor in the benign adenomatous stage, PTC is the epitheliomatous stage, and trichilemmal carcinoma is the carcinomatous stage.

Here, we report a very rare case of a trichilemmal cyst that underwent this series of changes. The development of trichilemmal carcinoma after PTC excision indicates the possibility of malignant transformation of the PTC.

\section{CASE REPORT}

A 27-year-old male patient presented at our institution with a hard 
mass on his posterior neck (Fig. 1). He reported that a mass $1 \times 1 \mathrm{~cm}$ in size had first been observed 7 years earlier. Incision and drainage (I\&D) of this mass, thought to be an epidermal cyst, had been performed 8 months prior to his current presentation. However, the size of the mass had gradually increased to approximately $5 \times 4 \mathrm{~cm}$

The patient was referred to the department of dermatology for punch biopsy, and the diagnosis was warty dyskeratoma. Eight months after $I \& D$, the lesion was excised without a safety margin, and a split-thickness skin graft was performed in our department. The final pathologic diagnosis was benign PTC. Histologic examination with hematoxylin and eosin (H\&E) staining showed welldedifferentiated pilar-type keratinization; the lesion was limited to the dermis without cellular atypia or mitotic activity (Fig. 2). The mass did not recur during a follow-up period of several months; however, the patient noticed a mass developing after 4 months. When he presented to our institution, discharge from the mass and surface ulceration were noted. Magnetic resonance imaging (MRI) revealed a newly developed $5 \times 3 \mathrm{~cm}$ mass that invaded the muscle layers (Fig. 3); therefore, surgical excision was planned.

Results of intraoperative frozen biopsy suggested trichilemmal carcinoma, requiring wide excision with a $1.3-\mathrm{cm}$ safety margin. The split-thickness skin graft (approximately $10 \times 8 \mathrm{~cm}$ ) was harvested from the right thigh with a Zimmer air-driven dermatome (Zimmer, Dover, OH, USA). The soft tissue defect of the posterior neck was covered with the skin graft and secured with 4-0 black

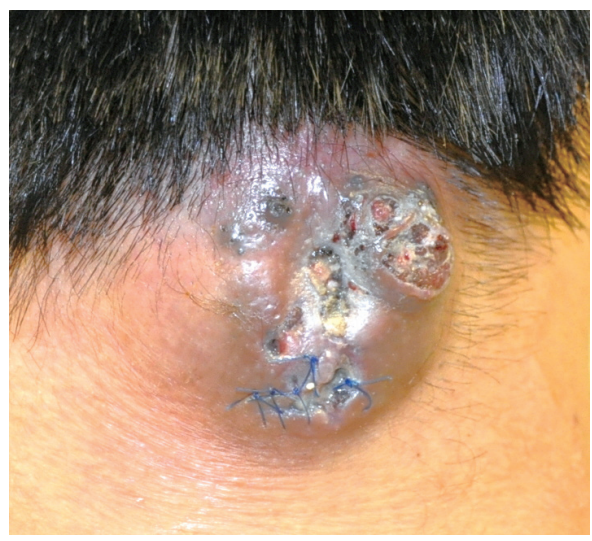

Fig. 1. Preoperative photograph before the first surgery reveals a cutaneous and subcutaneous enhancing solid tumor-like lesion $(2.5$ $\mathrm{cm}$ deep, $4 \mathrm{~cm}$ wide) in the right posterior neck, not a cystic lesion. silk sutures. The final pathologic diagnosis was trichilemmal carcinoma arising from PTC. Histologic analysis of the trichilemmal carcinoma showed marked atypia, pleomorphism, and invasion of the surrounding connective tissue (Fig. 4).

After the second surgery, radiotherapy was initiated on postoperative day 43 (planned dose: 5,760 cGy total, 180 cGy/treatment). The patient underwent 32 radiotherapy treatments over the course of 45 days without complications. Follow-up MRI 5 months after the second operation showed no evidence of remnant or recurrent cancer and no abnormal lymph node enlargement. A chemotherapy regimen used for squamous cell carcinoma (5-fluorouracil and cisplatin) was initiated, and the four cycles were completed 8 months after the second operation.

At postoperative year 1 following the second operation, positron emission tomography (PET) and MRI scans revealed no specific findings relevant to carcinoma. Follow-up MRI scans (every 6 months) found no recurrence or abnormalities.

\section{DISCUSSION}

PTC is an uncommon lesion characterized by the presence of trichilemmal keratinization. The characteristic histologic feature of trichilemmal keratinization is abrupt transition of the nucleated epithelial cells to keratinized cells without formation of a granular layer. The pattern is similar to that of the outer root sheath of
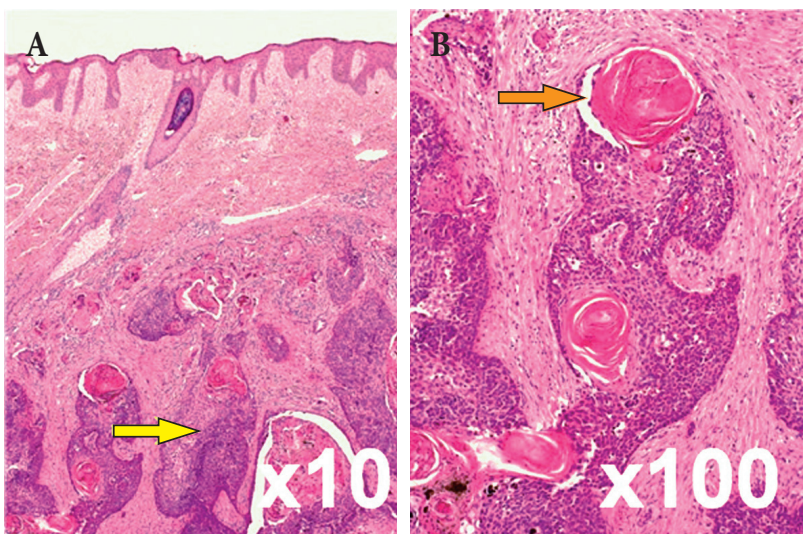

Fig. 2. (A, B) Histopathologic examination of the initial excisional biopsy shows that the proliferating trichilemmal cyst is limited to the dermal layer (yellow arrow) without cellular atypia or mitotic activity, containing welldedifferentiated pilar-type keratinization (orange arrow) (H\&E). 

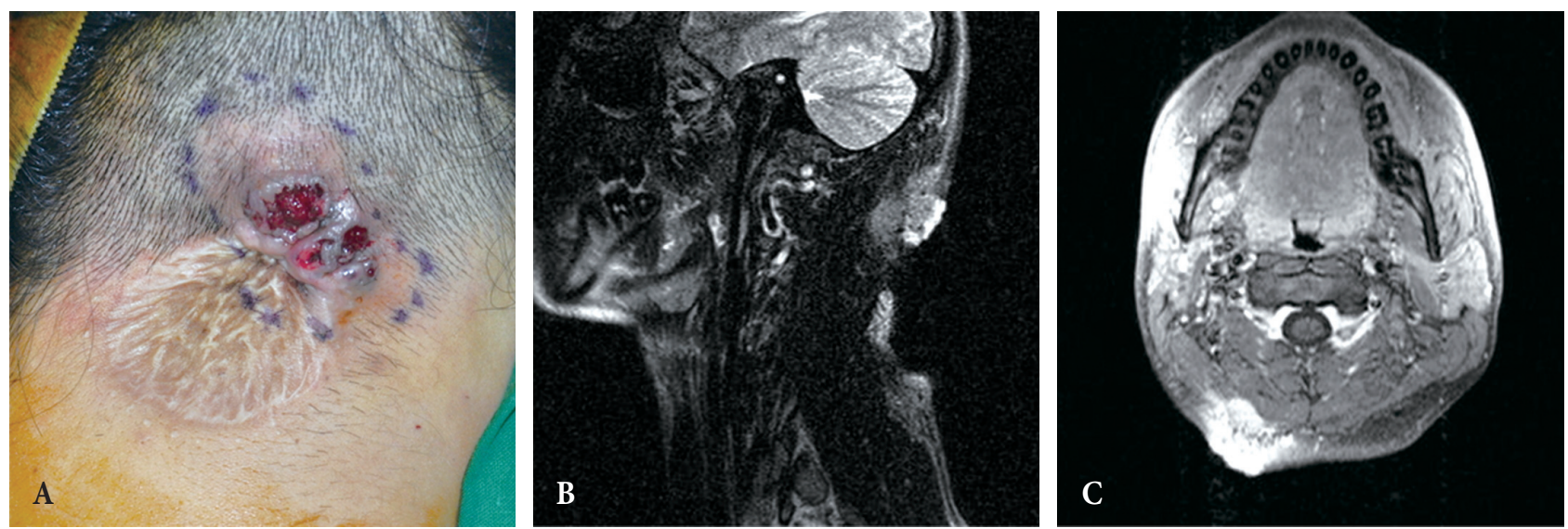

Fig. 3. (A) Preoperative photograph before the second surgery. (B, C) T2-weighted magnetic resonance imaging scan before the second surgery reveals a partially well-defined and partially infiltrative enhancing soft tissue mass with a bulging contour $(4 \mathrm{~cm}$ wide, $2.2 \mathrm{~cm}$ deep) in the cutaneous and subcutaneous layers of the right posterior neck. Close contact with or superficial invasion of the right posterior neck muscle is observed.
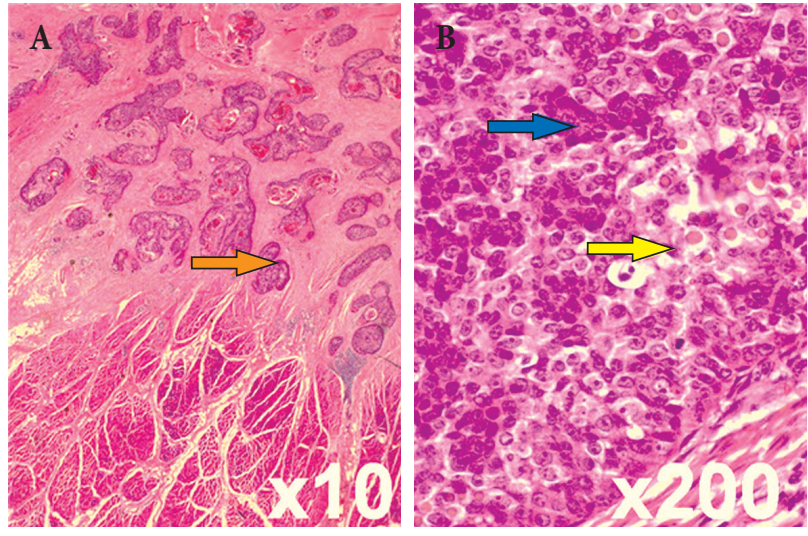

Fig. 4. (A, B) Histopathologic examination after the second surgery (wide excision) reveals a trichilemmal carcinoma with deep muscular invasion (orange arrow), cellular atypia (blue arrow), and mitotic activity (yellow arrow) (H\&E).

a hair follicle [6]. Sporadic intraepithelial keratinization sometimes results in small globules of dense keratin that compress adjacent cells. Varying levels of inflammation can be present, as indicated by lymphocyte, histiocyte, plasma cell levels, and occasionally neutrophil and eosinophil levels. The degree of cellularity, atypia, and nuclear pleomorphism also varies. Most tumors with mild or no atypia have 0 to 4 mitoses per 10 high-power fields. In this case, the pathologic slide of the first excisional biopsy showed well-differentiated pilar type keratinization (Fig. 2, orange arrow). The lesion was limited to the dermal layer, and no cellular atypia or mitotic activity was observed.

Since trichilemmal carcinoma is an adnexal tumor, its most common location on the head and neck regions may be explained by the higher concentration of skin appendages in these areas [7]

The diagnosis of trichilemmal carcinoma can be made in the presence of abnormal mitoses, high mitotic rate, marked cellular pleomorphism, cytologic and architectural atypia, infiltrating margin, necrosis, and aneuploidy. However, tumors with little or no cytologic or architectural atypia can be aggressive, and vice versa $[8,9]$. In some cases, the PTC contains an area indistinguishable from trichilemmal carcinoma [10]. Takata et al. [11] suggested that the critical event responsible for progression from the pre-existing PTC to trichilemmal carcinoma may be complete loss of the wild-type p53 tumor suppressor gene due to allelic loss of the short arm of chromosome 17p. In the present case, after the second surgery, the pathology slide revealed that the mass had invaded muscle layers and showed numerous abnormal mitoses and marked cellular pleomorphism (Fig. 4). Keratin pearl formation was also noted.

Current staging of adnexal carcinoma is included in the American Joint Committee on Cancer cutaneous squamous cell carcinoma staging. As is the case for many rare tumors, there is no standard of treatment.

Treatment of a PTC is simple excision; however, for carcinoma, wide excision with a 1-cm safety margin is recommended to prevent recurrence. Although surgical excision is considered the first choice for curative treatment, in some cases ethanol injection, 
lymph node dissection, radiotherapy, and chemotherapy can be considered [12].

Surgical excision followed by adjuvant radiotherapy is often performed in select cases [13].

Pugh et al. [13] reported that patients receiving postoperative RT (PORT) show improved clinical regression, and the use of PORT should be considered in patients with high-risk features for local recurrence.

In the present case, we concluded that the tumor had an aggressive nature, and conducted PORT in consultation with the oncology department.

Differential diagnoses include squamous cell carcinoma, basal cell carcinoma, keratoacanthoma, pilomatrixoma, dermatofibrosarcoma protuberans, cylindroma, angiosarcoma, and epidermoid cyst. Recurrence of PTC has been reported, but the transformation of PTC to trichilemmal carcinoma is rare. The rate of local recurrence is $3.7 \%$, with time of recurrence ranging from 6 months to more than 10 years $[14,15]$. Here we report a case of rare transformation of trichilemmal cyst to trichilemmal carcinoma via PTC.

Several studies suggest that PTC may have a greater malignant potential than histologically similar cutaneous squamous cell carcinomas [14]. In addition, trichilemmal carcinoma may exhibit aggressive invasion, even intracranially, causing considerable morbidity and even mortality [6]. Therefore, examination of patients with trichilemmal cyst should take into account the possibility of malignant transformation. Close follow-up is necessary for early identification of recurrence.

\section{REFERENCES}

1. Brownstein MH, Arluk DJ. Proliferating trichilemmal cyst: a simulant of squamous cell carcinoma. Cancer 1981;48:1207-14.

2. Saida T, Oohara K, Hori Y, Tsuchiya S. Development of a malignant proliferating trichilemmal cyst in a patient with multiple trichilemmal cysts. Dermatologica 1983;166:203-8.

3. Markal N, Kurtay A, Velidedeoglu H, Hucumenoglu S. Malignant transformation of a giant proliferating trichilemmal tumor of the scalp: patient report and literature review. Ann Plast Surg 1998;41:3146.

4. Yamaguchi J, Irimajiri T, Ohara K. Proliferating trichilemmal cyst arising in the arm of a young woman. Dermatology 1994;189:90-2.

5. Casas JG, Woscoff A. Giant pilar tumor of the scalp. Arch Dermatol 1980;116:1395.

6. Satyaprakash AK, Sheehan DJ, Sangueza OP. Proliferating trichilemmal tumors: a review of the literature. Dermatol Surg 2007;33:1102-8.

7. Laochumroonvorapong P, Kokta V, Quan MB. Trichilemmal carcinoma in an African American. Dermatol Surg 2002;28:284-6.

8. Noto G, Pravata G, Arico M. Malignant proliferating trichilemmal tumor. Am J Dermatopathol 1997;19:202-4.

9. Jaworski R. Malignant trichilemmal cyst. Am J Dermatopathol 1988;10:276-7.

10. Rutty GN, Richman PI, Laing JH. Malignant change in trichilemmal cysts: a study of cell proliferation and DNA content. Histopathology 1992;21:465-8.

11. Takata M, Rehman I, Rees JL. A trichilemmal carcinoma arising from a proliferating trichilemmal cyst: the loss of the wild-type 553 is a critical event in malignant transformation. Hum Pathol 1998;29:193-5.

12. Hayashi I, Harada T, Muraoka M, Ishii M. Malignant proliferating trichilemmal tumour and CAV (cisplatin, adriamycin, vindesine) treatment. Br J Dermatol 2004;150:156-7.

13. Pugh TJ, Lee NY, Pacheco T, Raben D. Microcystic adnexal carcinoma of the face treated with radiation therapy: a case report and review of the literature. Head Neck 2012;34:1045-50.

14. Ye J, Nappi O, Swanson PE, Patterson JW, Wick MR. Proliferating pilar tumors: a clinicopathologic study of 76 cases with a proposal for definition of benign and malignant variants. Am J Clin Pathol 2004;122:566-74

15. Mathis ED, Honningford JB, Rodriguez HE, Wind KP, Connolly MM, Podbielski FJ. Malignant proliferating trichilemmal tumor. Am J Clin Oncol 2001;24:351-3. 